\title{
Subspecialty Virtual Impact Factors within a Dedicated Neuroimaging Journal
}

\author{
(iD) A.F. Choudhri and (D) M. Castillo
}

\begin{abstract}
BACKGROUND AND PURPOSE: The growing number of subspecialties within neuroradiology compete for pages in neuroradiology journals. We performed a bibliometric analysis of the American Journal of Neuroradiology to identify the virtual Impact Factor of different journal subsections and article topics.
\end{abstract}

MATERIALS AND METHODS: Original Research and Review Articles published in American Journal of Neuroradiology during 2010-2012 were evaluated. The journal section for each article was recorded, and the number of citations was evaluated by using the Web of Science database. Numbers of citations within the first 2 years after publication were evaluated, normalized to the 2013 journal Impact Factor (for American Journal of Neuroradiology, 3.675), and used to calculate a virtual Impact Factor for different journal subsections.

RESULTS: One thousand forty-nine Original Research and Review Articles were published during this time, which obtained an average of 6.59 citations each within their first 2 years after publication; $91.8 \%$ of articles obtained at least 1 citation. Expedited Publications had the greatest number of citations, averaging 43.7 citations each (virtual Impact Factor, 24.39), followed by Review Articles averaging 9.39 citations each (virtual Impact Factor 5.23). Virtual Impact Factors for other sections were the following: Interventional, 4.54; Brain, 3.70; Pediatrics, 2.91; Functional, 2.74; Head \& Neck, 2.24; and Spine, 1.86. Virtual Impact Factors for article topics were the following: interventional, 4.75; functional/advanced, 3.79; brain, 3.66; pediatrics, 2.99; head and neck, 2.46; and spine, 2.32.

CONCLUSIONS: Citation patterns of Original Research and Review Articles in American Journal of Neuroradiology varied widely on the basis of subsections. Understanding the citation patterns of specific topics and subsections of a journal may aid authors and editors in evaluating the appropriate balance among various topics and allow authors to determine whether their articles are being cited at a level expected for similar ones in a journal.

ABBREVIATIONS: JIF = journal Impact Factor; VIF = virtual Impact Factor

C tation analysis is an important means of evaluating the performance of journals and of authors, with implications regarding promotions, grant funding, advertising sales, and library selections. ${ }^{1-3}$ The growing number of subspecialties within neuroradiology compete for journal pages, and citation profiles of

Received February 4, 2015; accepted after revision March 19

From the Departments of Radiology (A.F.C.), Neurosurgery (A.F.C.), and Ophthalmology (A.F.C.), University of Tennessee Health Science Center, Memphis, Tennessee; Department of Radiology (A.F.C.), Le Bonheur Children's Hospital, Memphis, Tennessee; and Department of Radiology (M.C.) University of North Carolina, Chapel Hill, North Carolina.

This work was supported by an AJNR Editorial Fellowship to A.F.C.

Paper previously presented at: American Society of Neuroradiology Annual Meeting and the Foundation of the ASNR Symposium, April 25-30, 2015; Chicago, Illinois.

Please address correspondence to Asim F. Choudhri, MD, Department of Radiology, Le Bonheur Children's Hospital, 848 Adams Ave-G216, Memphis, TN 38103;

e-mail: achoudhri@uthsc.edu; @AsimChoudhriMD

http://dx.doi.org/10.3174/ajnr.A4380 these different subsections have not been evaluated. We performed a bibliometric analysis of American Journal of Neuroradiology $(A J N R)$ to identify citation patterns based on subsections and article topics.

Neuroradiology encompasses evaluation of the brain, head and neck, spine, and peripheral nervous system, including minimally invasive diagnostic and therapeutic techniques in these areas. Several journals dedicated to neuroradiology exist; however, neuroradiologic articles are also included in general radiology journals and in radiology subspecialty journals such as those dedicated to pediatric radiology. While there is a growing body of bibliometric analyses in the radiology literature, ${ }^{4-7}$ few studies have focused on neuroradiology topics to date. ${ }^{8-10}$

We hypothesized that the citation characteristics of a journal are heterogeneous among journal subsections and varying topics. We undertook a bibliometric analysis to evaluate the citation characteristics of neuroradiology articles within a neuroradiology 
Table 1: Citation characteristics of journal sections (2010-2012) based on the first 2 years of citations

\begin{tabular}{lrrrrrrrrr}
\hline \multicolumn{1}{c}{\begin{tabular}{c} 
Journal \\
\multicolumn{1}{c}{ Category }
\end{tabular}} & No. & Mean & SD & Minimum & Maximum & Median & $\begin{array}{c}\text { \% with Citations } \\
\text { in First 2 Years }\end{array}$ & VIF \\
\hline Expedited Pub & 11 & 43.73 & 31.94 & 13 & 116 & 36 & 1.000 & 24.38 \\
Review & 53 & 9.38 & 7.95 & 0 & 38 & 7 & 0.981 & 5.23 \\
Interventional & 232 & 8.13 & 9.91 & 0 & 83 & 5 & 0.948 & 4.54 \\
Brain & 321 & 6.64 & 6.18 & 0 & 61 & 5 & 0.941 & 3.70 \\
Extracranial & 9 & 6.33 & 5.96 & 0 & 19 & 5 & 0.889 & 3.53 \\
$\quad$ Vascular & & & & & & & & \\
Pediatrics & 98 & 5.22 & 4.99 & 0 & 36 & 4 & 0.949 & 2.91 \\
Other $^{2}$ & 68 & 5.12 & 6.18 & 0 & 31 & 3 & 0.868 & 2.85 \\
Functional & 34 & 4.91 & 3.75 & 0 & 17 & 4 & 0.941 & 2.74 \\
Head \& Neck & 131 & 4.02 & 4.63 & 0 & 34 & 3 & 0.824 & 2.24 \\
Spine & 92 & 3.33 & 3.13 & 0 & 15 & 3 & 0.848 & 1.85 \\
Noncategorized $^{\mathrm{b}}$ & 77 & 1.91 & 3.92 & 0 & 19 & 0 & 0.403 & 1.06 \\
\hline
\end{tabular}

a Other includes Patient Safety $(n=31)$, Pharmacology Vignette $(n=10)$, Research Perspectives $(n=9)$, Practice Perspectives ( $n=7)$, Health Care Reform Vignette $(n=6)$, and Methodologic Perspective $(n=5)$.

b While the citation characteristics for these noncategorized items (editorials, letters to the editor, and so forth) are
Note:-Pub indicates Publication. presented here, they were not considered in the formula for determining the VIF correction factor.

Citations from noncategorized items, including letters to the editor, editorials, and errata, were not included in the VIF calculation. Each article selected for analysis was reviewed in the Web of Science database to determine how many citations it received. While we could find no existing bibliometric reports formally documenting this methodology, we believe that using the average number of citations for each journal subsection is a valid extension of the reported methodology of the JIF. ${ }^{11-14}$

\section{Statistics}

Data were collected in a spreadsheet (Excel; Microsoft, Redmond, Washington), and statistics were calculated by using SPSS Version 21 (IBM, Armonk, New York) and Excel. Discrete variables journal. We additionally sought to determine the citation characteristics of neuroradiology articles pertaining to specific subtopics.

\section{MATERIALS AND METHODS Article Selection}

Only Original Research and Review Articles published in AJNR from 2010 to 2012 were evaluated. Full-text versions of all articles were obtained as PDF downloads and reviewed for topic. This study did not directly involve patient information and met the criteria for institutional review board exemption.

\section{Journal Section Categorization}

The journal section for each article (eg, Brain, Head \& Neck, Pediatrics, Spine, Functional) was recorded. Additional journal subcategorizations, such as "Fellows' Journal Club" or "Editor's Choice" were also recorded.

\section{Article Topic Categorization}

The PDF of each article was evaluated to identify included topics, regardless of the initial categorization by the journal. While an article has only 1 category, as designated in the journal, there may be more than 1 topic that applies. For example, an article in the Pediatrics section of the journal on a head and neck topic will be assigned a topic of both pediatrics and head and neck, and a spine intervention article in the Interventional section would be assigned to the spine and interventional topics.

\section{Citation Analysis}

Each selected article was evaluated by using the Web of Science (Thompson Reuters, New York, New York) database for its number of citations. The number of citations within the first 2 calendar years after their publication was recorded and normalized to the 2013 AJNR journal Impact Factor (JIF, 3.675). This ratio was used to calculate a virtual Impact Factor (VIF) for each journal subsection. For instance, if a journal has a JIF of 5 , and the articles received, on average, 8 citations, then a journal subsection that received an average of 4 citations would have a VIF of 2.5. (number of articles with zero versus greater than 1 citation) were compared using the Fisher exact test. Continuous variables (number of citations, VIF) were compared with a Student $t$ test when there was a Gaussian distribution of data and otherwise with the nonparametric Wilcoxon rank sum test. $P$ values $<.05$ were considered significant.

\section{RESULTS}

One thousand forty-nine Original Research and Review Articles were published in AJNR during the 3 years evaluated. An additional 77 uncategorized items, including editorials, letters to the editor, and errata, were excluded from analysis. The 1049 categorized articles accumulated $6.59 \pm 8.53$ (range, $0-116$; median, 4) citations within their first 2 years after publication, and $91.8 \%$ of articles had at least 1 citation. On the basis of a JIF of 3.675, the VIF for a given section was determined as (Number of Citations / (6.59 / 3.675).

\section{Citations by Journal Section}

Expedited Publications had the most citations with 11 articles averaging $43.7 \pm 31.9$ (range, 13-116; median, 36) citations each (VIF, 24.39). Review Articles had the next highest number of citations, with 53 articles averaging $9.38 \pm 7.95$ (range, 0 -38; mereceived at least 1 citation within 2 years of publication. VIFs for other sections ranged from 4.54 for Interventional (the highest) to 1.86 for Spine (the lowest) (Table 1). Articles selected as "Editor's Choices" had a VIF of 5.14, and those selected for the "Fellows' Journal Club" had a VIF of 3.83 .

\section{Citations by Article Topic}

When we evaluated the citation characteristics by article topic as opposed to journal-assigned categories, interventional articles had the highest citation numbers, averaging $8.52 \pm 12.54$ (range, $0-116$; median, 5) citations with a VIF of 4.75 , which was similar to the journal category of Interventional $(P=.69)$ (Table 2). Articles related to the spine were cited $4.16 \pm 3.69$ (range, $0-19$; dian, 7) citations each (VIF, 5.23), and 52 (98.1\%) of these articles 
Table 2: Citation characteristics by topic and other designations

\begin{tabular}{|c|c|c|c|c|c|c|c|c|}
\hline Topic & No. & Mean & SD & Minimum & Maximum & Median & $\begin{array}{l}\% \text { with Citations } \\
\text { in First } 2 \text { Years }\end{array}$ & VIF \\
\hline Interventional & 317 & 8.52 & 12.54 & 0 & 116 & 5 & 0.927 & 4.753 \\
\hline Functional/advanced & 199 & 6.80 & 5.49 & 0 & 36 & 6 & 0.965 & 3.791 \\
\hline Brain & 484 & 6.57 & 6.28 & 0 & 61 & 5 & 0.940 & 3.663 \\
\hline Pediatrics & 115 & 5.36 & 5.12 & 0 & 36 & 4 & 0.930 & 2.987 \\
\hline Head and neck & 189 & 4.42 & 4.46 & 0 & 34 & 3 & 0.868 & 2.463 \\
\hline Spine & 145 & 4.16 & 3.69 & 0 & 19 & 3 & 0.883 & 2.319 \\
\hline Editor's Choice ${ }^{a}$ & 83 & 9.22 & 9.75 & 0 & 61 & 6 & 0.976 & 5.139 \\
\hline Fellows' Journal Club ${ }^{\mathrm{a}}$ & 82 & 6.87 & 5.72 & 0 & 31 & 6 & 0.902 & 3.828 \\
\hline $\begin{array}{l}\text { Nonvascular } \\
\text { interventional }\end{array}$ & 122 & 6.07 & 5.78 & 0 & 34 & 4.5 & 0.918 & 3.387 \\
\hline Peds head and neck & 17 & 4.94 & 3.42 & 0 & 11 & 4 & 0.941 & 2.755 \\
\hline Spine interventional & 64 & 4.14 & 3.37 & 0 & 15 & 7 & 0.891 & 2.309 \\
\hline
\end{tabular}

Note:-Peds indicates Pediatics.

a "Fellows' Journal Club" and "Editor's Choices" are journal designations that are independent of the journal-selection category.

median, 3) times, with a VIF of 2.32, which was higher than the Spine category of the journal $(P=.074)$. The percentage of articles having at least 1 citation within 2 years of publication ranged from $82.4 \%$ for Head \& Neck articles (108 of 131) to 100\% for Expedited Publication (11 of 11).

\section{Number of Articles Receiving at Least 1 Citation}

The number of publications receiving at least 1 citation within 2 years was statistically lower for the Head \& Neck category compared with Review Articles $(P=.003)$, Interventional $(P=.0003)$, Brain $(P=.0003)$, and Pediatrics $(P=.0042)$. The number of publications receiving at least 1 citation within 2 years was statistically lower for the Spine category than Review Articles $(P=$ $.0103)$, Interventional $(P=.0053)$, Brain $(P=.0075)$, and Pediatrics $(P=.028)$.

\section{DISCUSSION}

Citation patterns of Original Research and Review Articles in AJNR varied widely on the basis of subsections, with Review Articles accumulating the greatest number of citations within the first 2 years after publication. Understanding these citation patterns for specific topics and subsections of a journal may aid editors in evaluating the appropriate balance between topics and allow authors to determine whether their articles are being cited at levels expected for similar topics in a journal.

The highest number of citations was for articles classified as "Expedited Publication," which is not unexpected because these are presumably important and timely topics. Thus, encouraging the submission of such articles has the potential to influence the Impact Factor of a journal. Expedited peer review and waived fees, such as those for color figures or article length, may be ways to encourage such submissions as AJNR does.

Articles that received the designation of "Editor's Choice" and "Fellows' Journal Club" were cited more often than others in their given journal categories. These designations are chosen by the Editor-in-Chief, and it is possible that the assigned designations improved recognition of the articles and resulted in increased citations. In addition, the articles chosen for these categories are usually those with state-of-the-art topics, clinically important, and of higher evidence-based levels, which may also contribute to their increased citations. Articles related to spine intervention trended toward a higher number of citations than diagnostic spine articles overall, probably because they are viewed and cited by specialists outside of just imaging.

Limitations in our study include the inability to determine the exact parameters that compose the official JIF because this is a proprietary algorithm that is modified with time. Additionally, the JIF changes with time, and we only used a single JIF for this investigation, the 2013 JIF, which was the most recent one available at the time of analysis. As with all citations, it is not possible to determine citations when the original article serves as a foundation for future work or when it is criticized or refuted. Many articles are well-received by readers, becoming popular reading but are not cited. One of the reasons many journals have decreased or eliminated case reports, despite their popularity among readers, is the negative effect on the JIF. We created our VIF on the basis of citations in the first 2 years after publication, mirroring the methodology of the JIF. The JIF also can be calculated for other periods of citations, in particular the 5-year JIF, which may give additional information about the longevity of impact of an article; however, we chose to perform a 2-year analysis because the 2-year JIF is the most commonly used metric.

The resultant quality of articles in a journal is a factor of the quality of work being performed in a given area, the alternative outlets for publication that a given author may choose, and the quality of the editorial review process. It is possible that areas such as interventional, in which many reviewers practice for a large percentage of their careers, receive higher quality reviews because the reviewers want to simultaneously select the best articles and provide the best feedback to the authors during the review process to improve the articles. In contrast, a smaller number of radiologists have most of their practice in head and neck and likely even fewer have their practice and academic interests primarily in spine.

Beyond neuroradiology journals, there are spine-focused journals in neurosurgy and orthopaedic surgery (eg, Journal of Neurosurgery-Spine, and Spine). These 2 journals have JIFs of 2.355 and 2.447 , respectively, very similar to the 2.319 VIF for articles with a spine topic in AJNR. Comparisons such as this may help determine whether subsections and/or topics are being cited like those in journals with a potentially overlapping focus.

Knowledge of subsection VIF may allow prediction of the influence on the JIF, with reallocation of journal pages between sections. Page allotment limitations from print journals may be mitigated as they shift toward on-line publications.

Because the financial and logistic barriers for creating larger new journals has decreased, it may be tempting for physicians with a narrow area of interest to contemplate creation of a focused journal on a given topic, and understanding citation trends may provide evidence that a narrowly focused journal may or may not have the ability to survive. For example, it is clear from our investigation that for subspecialties such as spine and head and neck 
imaging, creating their own freestanding journals may be difficult and physicians are better served by remaining within a larger journal with a higher JIF than they would in independent journals dedicated to their specialty. In addition, authors of articles with a given topic may be able to better recognize the expected citations of their article and whether submitting to a specific journal such as $A J N R$ is best for them. For instance, the author of a diagnostic spine imaging article in AJNR, which will have fewer citations than the average article in the journal, may be less discouraged if they know their article has been cited more than other diagnostic spine articles in the same or other journals. Thus, encouraging all journals to calculate and publish their virtual JIF by sections may be useful to prospective authors.

\section{CONCLUSIONS}

Understanding the citation expectations of specific topics and subsections of a journal may aid editors in evaluating the appropriate balance among various topics and allow authors to determine whether their article is being cited at a level expected for similar topics in a journal.

\section{REFERENCES}

1. Chew FS, Relyea-Chew A. How research becomes knowledge in radiology: an analysis of citations to published papers. AJR Am J Roentgenol 1988;150:31-37

2. Chew FS. The scientific literature in diagnostic radiology for American readers: a survey and analysis of journals, papers, and authors. AJR Am J Roentgenol 1986;147:1055-61
3. Choudhri AF, Siddiqui A, Khan NR, et al. Understanding bibliometric paramaters and analysis. Radiographics 2015;35:736-46

4. Yoon DY, Yun EJ, Ku YJ, et al. Citation classics in radiology journals: the 100 top-cited articles, 1945-2012. AJR Am J Roentgenol 2013;201:471-81

5. Brinjikji W, Klunder A, Kallmes DF. The $\mathbf{1 0 0}$ most-cited articles in the imaging literature. Radiology 2013;269:272-76

6. Pagni M, Khan NR, Cohen HL, et al. Highly cited works in radiology: the top 100 cited articles in radiologic journals. Acad Radiol 2014;21:1056-66

7. Yun EJ, Yoon DY, Kim BY. Where do radiologists publish their work? A comparative analysis of publications by radiologists in nonradiology journals in 2000 and 2010. AJR Am J Roentgenol 2013;200:W560-65

8. McDonald RJ, Cloft HJ, Kallmes DF. Fate of manuscripts previously rejected by the American Journal of Neuroradiology: a follow-up analysis. AJNR Am J Neuroradiol 2009;30:253-56

9. Khosla A, McDonald RJ, Bornmann L, et al. Getting to yes: the fate of neuroradiology manuscripts rejected by Radiology over a 2-year period. Radiology 2011;260:3-5

10. Lee JY, Yoon DY, Yoon SD, et al. Neurointerventional research between 2003 and 2012: slow growth, high interdisciplinary collaboration, and a low level of funding. AJNR Am J Neuroradiol 2014;35:1877-82

11. Garfield E. The history and meaning of the journal Impact Factor. JAMA 2006;295:90-93

12. Garfield E. Citation analysis as a tool in journal evaluation. Science 1972:178:471-79

13. Garfield E, Cawkell AE. Citation analysis studies. Science 1975: 189:397

14. Garfield E. Citation indexes for science; a new dimension in documentation through association of ideas. Science 1955:122:108-11 